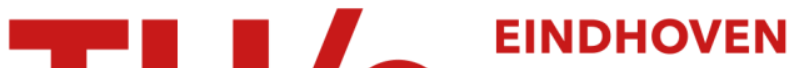 UNIVERSITY OF TECHNOLOGY
}

\section{Experimental observation of a hydrodynamic mode in a flow duct with a porous material}

Citation for published version (APA):

Aurégan, Y., \& Singh, D. K. (2014). Experimental observation of a hydrodynamic mode in a flow duct with a porous material. Journal of the Acoustical Society of America, 136(2), 567-572.

https://doi.org/10.1121/1.4884768

DOI:

10.1121/1.4884768

Document status and date:

Published: 01/01/2014

Document Version:

Publisher's PDF, also known as Version of Record (includes final page, issue and volume numbers)

Please check the document version of this publication:

- A submitted manuscript is the version of the article upon submission and before peer-review. There can be important differences between the submitted version and the official published version of record. People interested in the research are advised to contact the author for the final version of the publication, or visit the $\mathrm{DOI}$ to the publisher's website.

- The final author version and the galley proof are versions of the publication after peer review.

- The final published version features the final layout of the paper including the volume, issue and page numbers.

Link to publication

\section{General rights}

Copyright and moral rights for the publications made accessible in the public portal are retained by the authors and/or other copyright owners and it is a condition of accessing publications that users recognise and abide by the legal requirements associated with these rights.

- Users may download and print one copy of any publication from the public portal for the purpose of private study or research.

- You may not further distribute the material or use it for any profit-making activity or commercial gain

- You may freely distribute the URL identifying the publication in the public portal.

If the publication is distributed under the terms of Article $25 \mathrm{fa}$ of the Dutch Copyright Act, indicated by the "Taverne" license above, please follow below link for the End User Agreement:

www.tue.nl/taverne

Take down policy

If you believe that this document breaches copyright please contact us at:

openaccess@tue.nl

providing details and we will investigate your claim. 


\title{
Experimental observation of a hydrodynamic mode in a flow duct with a porous material
}

\author{
Yves Aurégan ${ }^{\text {a) }}$ \\ Laboratoire d'Acoustique de l'Université du Maine, Unité Mixte de Recherche 6613 Centre National de la \\ Recherche Scientifique, Avenue O Messiaen, F-72085 Le Mans Cedex 9, France \\ Deepesh Kumar Singh \\ Department of Mathematics and Computer Science, Eindhoven University of Technology, \\ $5600 \mathrm{MB}$ Eindhoven, The Netherlands
}

(Received 12 March 2014; revised 3 June 2014; accepted 9 June 2014)

\begin{abstract}
This paper experimentally investigates the acoustic behavior of a homogeneous porous material with a rigid frame (metallic foam) under grazing flow. The transmission coefficient shows an unusual oscillation over a particular range of frequencies which reports the presence of an unstable hydrodynamic wave that can exchange energy with the acoustic waves. This coupling of acoustic and hydrodynamic waves becomes larger when the Mach number increases. A rise of the static pressure drop in the lined region is induced by an acoustic excitation when the hydrodynamic wave is present. (C) 2014 Acoustical Society of America. [http://dx.doi.org/10.1121/1.4884768]
\end{abstract}

PACS number(s): 43.28.Py, 43.50.Gf, 43.20.Mv [AH]

Pages: 567-572

\section{INTRODUCTION}

Porous absorbers are used in many situations to achieve the absorption of sound, e.g., in ventilation ducts, in power plants, and in the exhaust systems of cars and trucks. It is a problem of high practical concern to obtain the largest attenuation of sound in a duct by using an appropriate acoustic treatment.

A satisfactory understanding of the acoustic behavior of homogeneous porous materials, in terms of fluid equivalent models, has been achieved by the current models. ${ }^{1-3}$ The benefits of the porous materials on the sound attenuation can be masked and even destroyed by the presence of a grazing flow. The thin flow boundary layer along the material plays a crucial role as it is involved in the interface condition between the propagation in air and the propagation in the porous material. Furthermore, this boundary layer may be unstable in the presence of a lined wall. This effect has been seen on locally reacting liners ${ }^{4}$ and optical measurements have shown that this instability is convective. ${ }^{5}$ This paper gives the experimental evidence of the existence of a hydrodynamic instability along the porous material triggered by the acoustic waves. The hydrodynamic waves are those that are convected in the flow direction and are nearly incompressible. These waves can be unstable over a liner. ${ }^{6}$ The presence of such hydrodynamic waves along a porous material has not been reported previously to our best knowledge. This paper only attends to demonstrate the existence of such waves. Further physical and mathematical investigations are needed to model this phenomena that can have important practical consequences by changing the transmission losses of porous absorbers.

This paper describes the behavior of a porous material with a rigid frame (metallic foam) under grazing flow. After

\footnotetext{
a) Author to whom correspondence should be addressed. Electronic mail: yves.auregan@univ-lemans.fr
}

a short description of the setup (Sec. II), the experimental scattering coefficients in a flow duct are presented (Sec. III). These coefficients show that an unstable hydrodynamic mode is present. The accessible characteristics of this hydrodynamic mode are extracted from the measurement of the scattering matrix (Sec. IV). The characteristics are compared with experimental results from 11 microphones located on the wall opposite to the porous material (Sec. V). Then the static pressure drop is measured with and without acoustic excitation of the material (Sec. VI).

\section{SETUP DESCRIPTION}

The test facility is schematically depicted in Fig. 1. Complete descriptions of this setup and of the used method have been reported previously ${ }^{4,7}$ and hence only the key points are presented here.

The setup allows acoustic propagation in a rectangular duct (width $A=100 \mathrm{~mm} \times$ height $W=15 \mathrm{~mm}$ ) superimposed

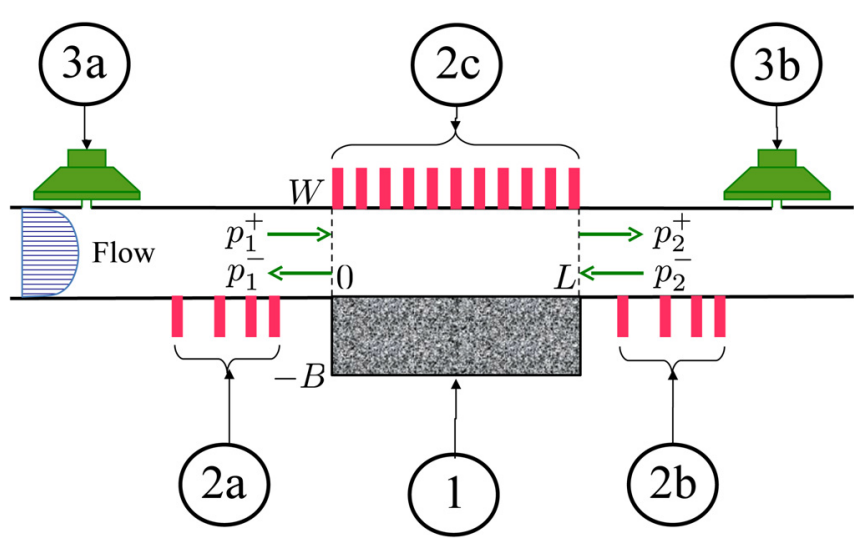

FIG. 1. (Color online) Schematic view of the experimental setup. 1: Porous material, 2a: four upstream microphones, $2 \mathrm{~b}$ : four downstream microphones, 2c: array of 11 microphones, 3a: upstream source, $3 \mathrm{~b}$ : downstream source (see Refs. 4 and 6 for details). 
to a grazing flow over an acoustic liner. The turbulent flow is assumed to be fully developed in the liner test section. At each end of the duct, acoustic source and anechoic termination are found. According to the duct dimensions and to the position of the microphones (at mid-width), only the plane waves are considered in the rigid parts of the duct for the frequency range of interest (100 to $3000 \mathrm{~Hz}$ ).

The scattering matrix for the plane waves relates the scattered pressure amplitudes $p_{1}^{+}$and $p_{1}^{-}$(see Fig. 1) to the incident pressure amplitudes $p_{1}^{+}$and $p_{2}^{-}$by

$$
\left(\begin{array}{c}
p_{2}^{+} \\
p_{1}^{-}
\end{array}\right)=\left[\begin{array}{ll}
T^{+} & R^{-} \\
R^{+} & T^{-}
\end{array}\right]\left(\begin{array}{l}
p_{1}^{+} \\
p_{2}^{-}
\end{array}\right),
$$

where $T^{+}$and $T^{-}$are the anechoic transmission coefficients, $R^{+}$and $R^{-}$are the anechoic reflection coefficients. The superscript "+" refers to an incident wave in the flow direction and the superscript "-." to an incident wave against the flow. This scattering matrix is measured by means of $2 \times 4$ flush mounted microphones in the lower hard wall downstream and upstream of the liner test section, respectively. It follows that the transmitted and reflected waves can be measured with over-determination in order to avoid inaccurate measurements when the acoustic wavelength is close to half the distance between two microphones. The two sources method is applied by doing two measurements where the upstream and downstream sources are switched on successively.

On the other hand, an array of eleven flush mounted microphones is located on the wall opposite to the porous material in order to measure the pressure evolution along the material. These microphones are evenly distributed along the $x$ axis spaced out by $2 \mathrm{~cm}$. Please note that the presence of these 11 microphones can affect the propagation in the treated region. This effect is supposed to be small and is disregarded in this paper.

The acquisition of signals is performed by Agilent VXI 1432 hardware platform which drives the source excitation synchronously with the acoustic pressure signals recording. A swept-sine over the frequency range $100-3000 \mathrm{~Hz}$ is used with a frequency increment of $5 \mathrm{~Hz}$. The amplitude of the excitation is automatically adapted to give a constant pressure, independently of the frequency, on the microphone just in front of the transition hard duct/material (on the upstream side when the upstream source is on and on the downstream side when the downstream source is on).

Experiments are carried out on a metallic foam (RECEMAT, NC4753.05 nickel-chromium alloy). This material has been chosen to be as rigid as possible in order to avoid any skeleton vibrations and was supplied as plates ( $L=200 \mathrm{~mm} \times A=100 \mathrm{~mm}$ ) of thickness $5 \mathrm{~mm}$. Five of these plates have been assembled with screws such that the total thickness of the material is $B=25 \mathrm{~mm}$. The parameters of this foam used in the fluid equivalent model have been measured on another setup. The values are porosity $\Phi=0.99$, tortuosity $\alpha_{\infty}=1.17$, viscous length $\Lambda=1 \times 10^{-4} \mathrm{~m}$, thermal length $\Lambda^{\prime}=2.4 \times 10^{-4} \mathrm{~m}$, resistivity $\sigma=6.9 \times 10^{3} \mathrm{~kg}$ $\mathrm{m}^{-3} \mathrm{~s}^{-1}$. Although this parameter is not very relevant here, the normal impedance of this porous material sample with a rigid backing is given in Fig. 2 for a comparison with locally

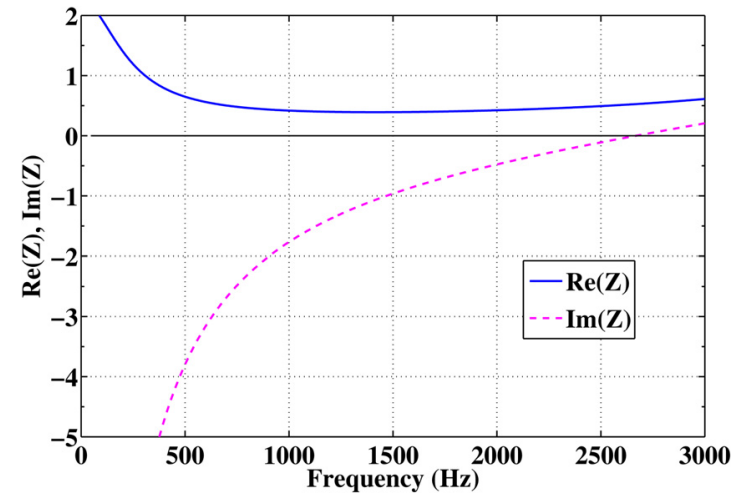

FIG. 2. (Color online) Real and imaginary part of the normal impedance of the porous material $(B=25 \mathrm{~mm})$ computed with the fluid equivalent model. The foam parameters are given in the text.

reacting liners. This impedance can be computed using the fluid equivalent $\operatorname{model}^{3}$ by $Z_{n}=-\mathrm{j}\left(Z_{\text {eq }}(f) / Z_{c}\right) /$ $\tan \left(k_{\mathrm{eq}}(f) B\right)$, where $Z_{\mathrm{eq}}(f)$ is the equivalent characteristic impedance of the porous material, $Z_{c}$ is the air characteristic impedance, and $k_{\mathrm{eq}}(f)$ is the equivalent wavenumber in the porous material. $Z_{\mathrm{eq}}$ and $k_{\mathrm{eq}}$ are frequency dependent.

\section{EXPERIMENTAL RESULTS FOR TRANSMISSION AND REFLECTION COEFFICIENTS}

The experimental results for transmission $\left(T^{+}\right.$and $\left.T^{-}\right)$ and the reflection $\left(R^{+}\right.$and $\left.R^{-}\right)$coefficients are given in Figs. 3 and 4, respectively. The curves in circles represent the results without flow. Due the reciprocity principle that exists without flow, the transmission coefficients are equal in both directions. The transmission coefficient for this case without flow decreases smoothly from lowest frequency up to the maximum measured frequency. The reflection coefficient oscillates about a value of $R_{0}=0.41$ which is close to the value $(1-\alpha) /(1+\alpha)=0.45$, where $\alpha=W /(W+B)$, valid at low frequencies for an area expansion without porous material. The oscillations in the reflection coefficient are linked to the wave reflection at the end of the material $(x=L)$. The reflection coefficients without flow are not strictly identical in both directions. The difference $(<10 \%)$ may be caused by

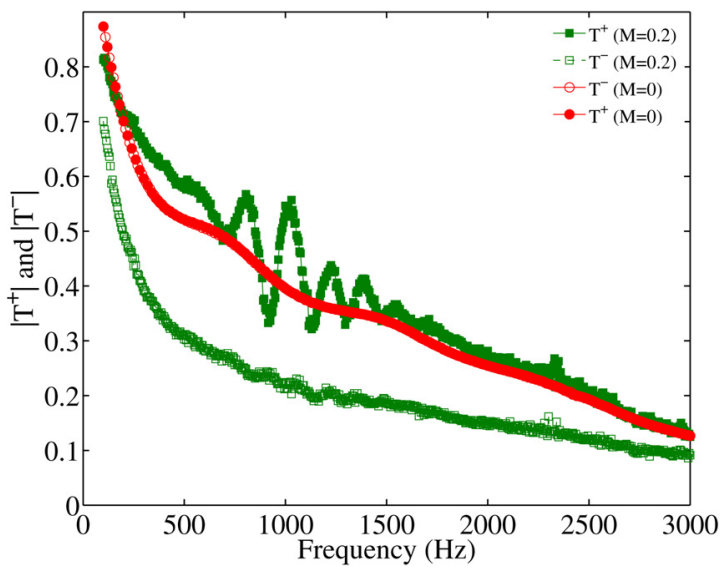

FIG. 3. (Color online) Absolute values of the transmission coefficients of the metallic foam sample without flow and for Mach number $M=0.2$. The source level is $134 \mathrm{~dB}$ SPL. 


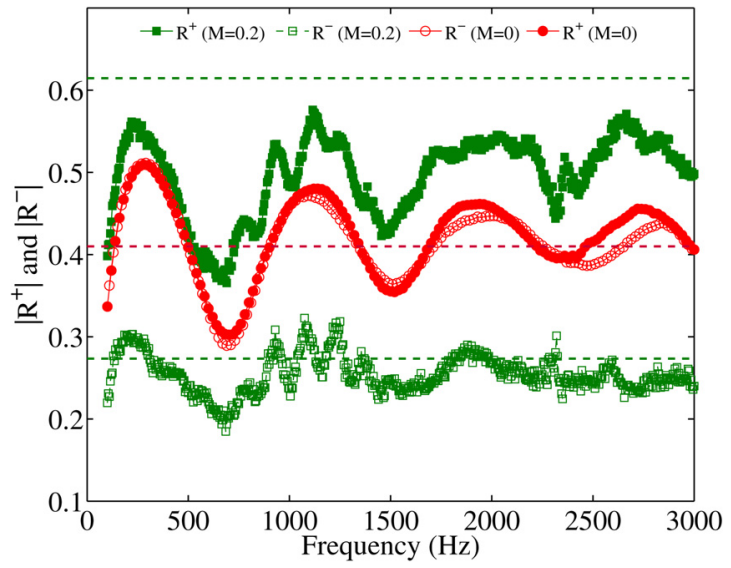

FIG. 4. (Color online) Absolute values of the reflection coefficients of the metallic foam sample without flow and for Mach number $M=0.2$. The dashed lines represent $R_{0}=0.41, R_{0}(1-M) /(1+M)$ and $R_{0}(1+M) /(1-M)$. The source level is $134 \mathrm{~dB}$ SPL.

some inhomogeneity of the material proprieties inducing a small breaking of symmetry.

The reflection coefficients for the waves propagating along the flow and against the flow differ significantly. The reflection coefficient against the flow $R^{-}$is close to the value $R^{-} \simeq R_{0}(1-M) /(1+M)$, where $M$ is the Mach number. The reflection coefficient in the flow direction $R^{+}$is between $R_{0}$ and $R_{0}(1+M) /(1-M)$ (see Fig. 4). The transmission coefficients are also different when the direction of wave propagation with respect to direction of flow is changed. Due to convection effects, the wavenumber increases when the wave propagates against the flow and the sound is more attenuated against the flow than in the case without flow. The overall attenuation in the flow direction is of the same order than the case without flow. Large oscillations in the frequency range $600-1600 \mathrm{~Hz}$ can be observed. It will be shown in the following that these oscillations result from the interference of the acoustic and hydrodynamic waves. The observation of these oscillations is the main result of this paper and will be studied in detail in the next section.

The measured transmission coefficient $T^{+}$(when the incident sound and the flow are in the same direction) is shown in Fig. 5 for two Mach numbers. The amplitude of the

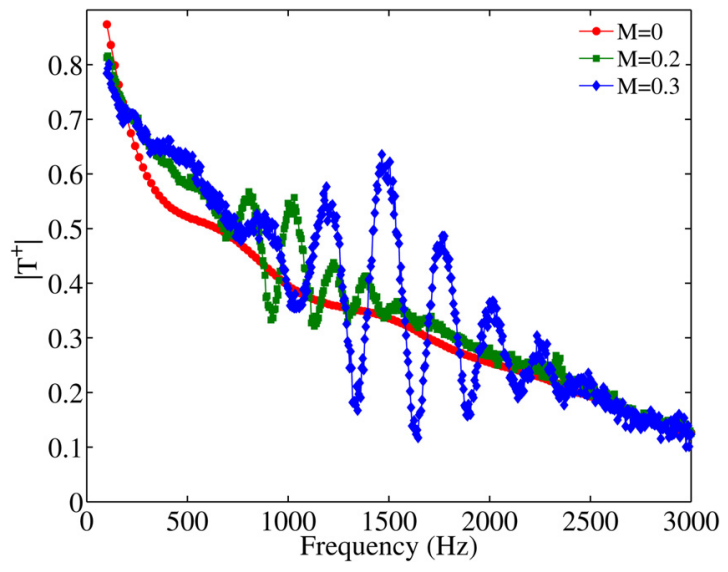

FIG. 5. (Color online) Plot of the measured transmission coefficient in flow direction $\left(T^{+}\right)$at different Mach numbers with the source level kept at $134 \mathrm{~dB}$ SPL. oscillations due to the interference of acoustic and hydrodynamic modes is higher for high Mach numbers. It is also interesting to see that the oscillations are prominent only in a certain frequency band which changes depending on the Mach number.

The amplitude of the oscillations changes with the sound source amplitude as well. Shown in Fig. 6 is the transmission coefficient $T^{+}$for three different upstream source amplitudes keeping the flow Mach number fixed at $M=0.3$. It can be observed that the amplitude of the oscillations of the absolute value of $T^{+}$increases when the source level decreases. It must be kept in mind that the oscillations in pressure is the product of $T^{+}$and the incident pressure. This value is increasing with the level but a clear saturation of the hydrodynamic effect can be observed.

\section{EXTRACTION OF THE HYDRODYNAMIC WAVE CHARACTERISTICS}

To fit the transmission coefficient in flow direction $T^{+}$, it can be seen as the sum of a contribution due to the "acoustic" transmission $T_{a}^{+}$and a contribution due to the "hydrodynamic" effects $T_{h}^{+}$. These two contributions propagate at different velocities and the net oscillations in $\left|T^{+}\right|$ can be seen as interferences between these two waves. The acoustic contribution is supposed to be smooth and hence $T_{a}^{+}$ is obtained by a fit of $T^{+}$in order to filter the oscillations. To obtain the better fit, $T_{a}^{+}$is searched under the form $T_{a}^{+}$ $=e^{-j k_{a} L}$ and the value of $k_{a}$ is approximated by a complex polynomial of degree 7 that fits the data best in a least-square sense. The result of the fit is displayed in Fig. 7. This fit gives good results because the effect of the acoustic wave propagating against the flow is weak. It should be noted that this fit includes all the entrance and exit effects and that $k_{a}$ is not the wave number of the least attenuated mode for $0<x<L$. Nevertheless, the real part of $k_{a}$ is linked to the velocity of the acoustic wave for $0<x<L$ because the entrance and exit effects can induce a small phase lag in the transmission coefficient. The equivalent acoustic velocity $c_{a}=\omega / k_{a}$ is shown in Fig. 7(b). Its value is around the sound velocity in air and takes into account the opposite effects of the convection (increase in the velocity) and of the porous material (slowing of the wave).

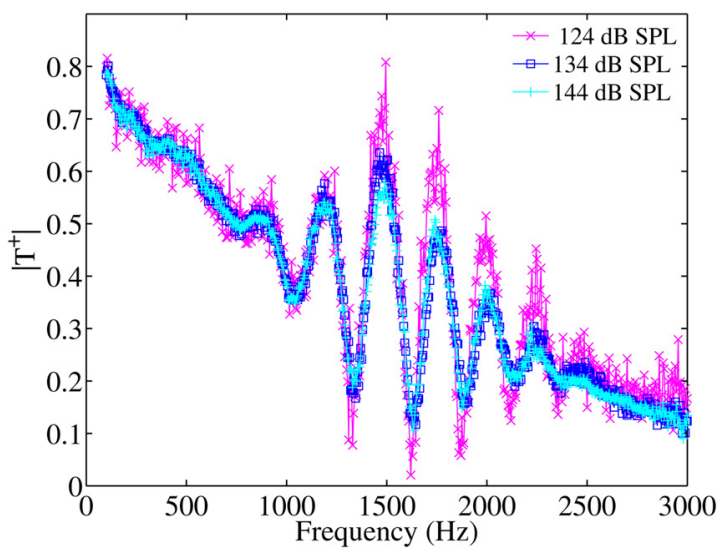

FIG. 6. (Color online) Plot of the measured transmission coefficient at different source amplitude with Mach number kept at $M=0.3$. 

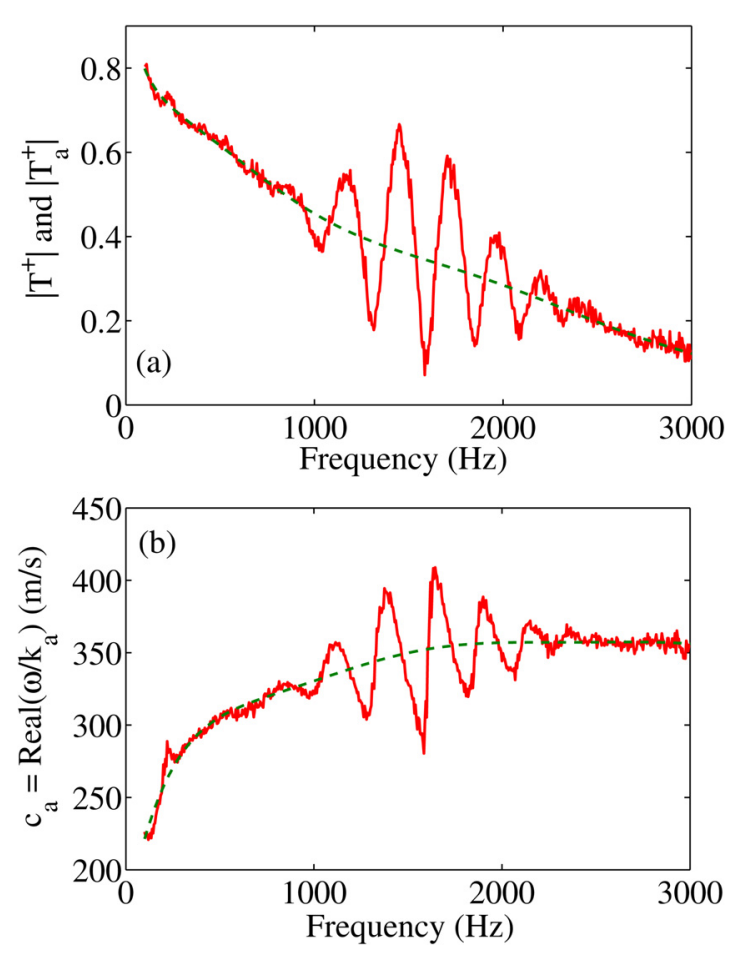

FIG. 7. (Color online) (a) Absolute value of $T^{+}$(continuous line) and of the fit $T_{a}^{+}$(dashed line). (b) Velocity of the acoustic wave computed by $c_{a}=\operatorname{Re}\left(\omega / k_{a}\right)$ (continuous line) and fit of the velocity (dashed line) $(M=0.3$ and $134 \mathrm{~dB}$ SPL).

The hydrodynamic contribution is obtained by subtracting the acoustic transmission $T_{a}^{+}$from the total transmission $T^{+}$. The amplitude and the phase of the hydrodynamic transmission $T_{h}^{+}$are given in Fig. 8. It can be seen that the hydrodynamic transmission can be sought under the form $T_{h}^{+}=H e^{-j\left(\theta_{0}+\omega L / c_{h}\right)}$, where $\omega=2 \pi f$ is the pulsation, $c_{h}$ is the velocity of the hydrodynamic wave, $\theta_{0}$ is the phase lag between the acoustic and the hydrodynamic waves and $H=\left|T_{h}^{+}\right|$is the amplitude of the effect of the hydrodynamic wave on the transmission coefficient called for simplicity in the following as "hydrodynamic wave amplitude."

The amplitude of the hydrodynamic transmission $\left|T_{h}^{+}\right|$is given in Fig. 8(a). It has a significant amplitude value on a limited frequency range (in this case 1000 to $2000 \mathrm{~Hz}$ ). A frequency $f_{m}$ at which the hydrodynamic mode has a maximum amplitude may be identified. This frequency depends on the Mach number but not on the level of the source. Shown in Table I are the obtained values of $f_{m}$ for different Mach numbers. On the other hand, the amplitude of this maximum depends on the Mach number as well as the source level. Thus, it is linked to the non-linear saturation of the hydrodynamic wave.

The angle of the hydrodynamic transmission $T_{h}^{+}$is given in Fig. 8(b). It is supposed that the slope of this angle is directly related to the velocity of the hydrodynamic wave. In Fig. 8(b), it can be seen that this velocity can be considered as constant in the frequency range of interest. The angle of $T_{h}^{+}$is then fitted by a straight line over the frequency range where the amplitude has a significant value. The value of the hydrodynamic wave velocity $c_{h}$ depends on Mach number but not on the incident sound pressure level (SPL). The value
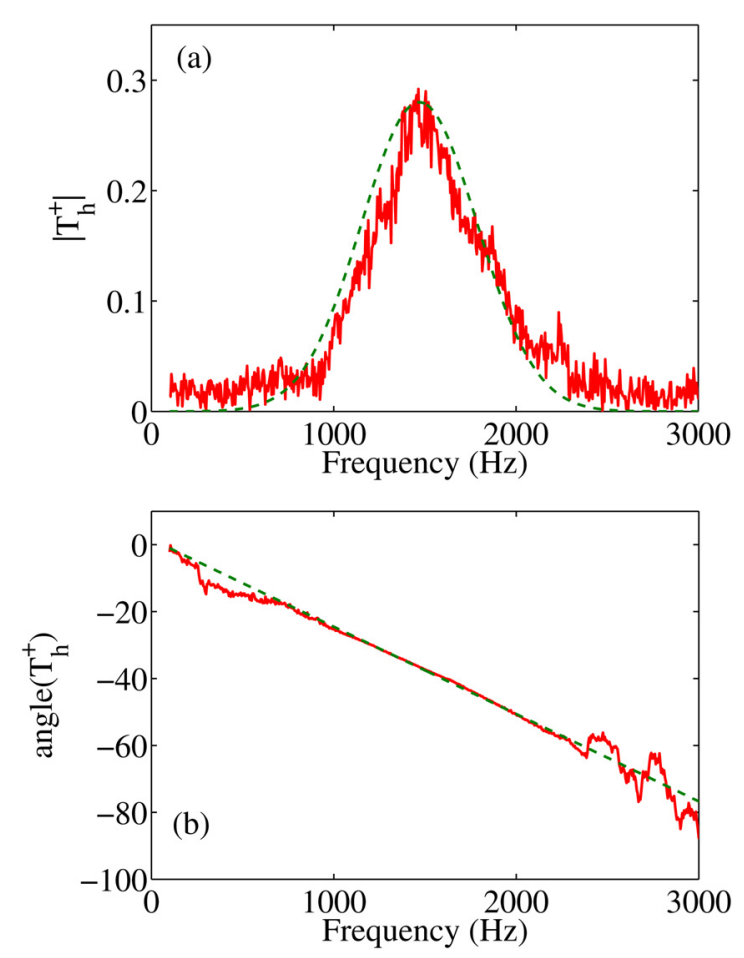

FIG. 8. (Color online) (a) Absolute value of $T_{h}^{+}$and (b) angle of $T_{h}^{+}$as a function of frequency ( $M=0.3$ and $134 \mathrm{~dB}$ SPL). The dashed line in (a) is the Gaussian function used to fit the amplitude curve. The dashed straight line in (b) is the linear approximation used to compute the velocity of the hydrodynamic wave.

of $M_{h}=c_{h} / c_{0}$, where $c_{0}$ is the sound velocity, is given in Fig. 9 as a function of the Mach number. It could be seen that the velocity of the hydrodynamic wave is nearly equal to half of the mean flow velocity. It can also be observed that the wavelength of the hydrodynamic perturbation for the maximum amplitude is nearly constant $\lambda_{h}=c_{h} / f_{m} \simeq 35 \mathrm{~mm}$ (see Table I).

Based on the above results, the following scenario can be imagined to explain the oscillating behavior of the transmission coefficient. An incoming acoustic wave from the upstream side of the porous material triggers a hydrodynamic wave on a limited range of frequencies. The acoustic wave propagates in the flow and in the material while the hydrodynamic wave is convected at half of the flow velocity. During the convection, the hydrodynamic wave is amplified and then its amplitude is saturated by non-linear effect. The interference between these two synchronized waves at the downstream side of the porous material induces oscillations in the transmission coefficient.

TABLE I. Velocity of the hydrodynamic wave $c_{h}$, frequency for maximum amplitude of the hydrodynamic mode $f_{m}$, hydrodynamic wave length $\lambda_{h}=c_{h} / f_{m}$ and frequency for maximum increasing of the pressure drop $f_{\mathrm{PD}}$ for various Mach numbers.

\begin{tabular}{lcccr}
\hline \hline$M$ & 0.15 & 0.2 & 0.25 & 0.3 \\
$c_{h}(\mathrm{~m} / \mathrm{s})$ & 30 & 35 & 44 & 49 \\
$f_{m}(\mathrm{~Hz})$ & 770 & 950 & 1220 & 1470 \\
$\lambda_{h}(\mathrm{~mm})$ & 39 & 37 & 36 & 33 \\
$f_{\mathrm{PD}}(\mathrm{Hz})$ & 1020 & 1280 & 1500 & 1820 \\
\hline \hline
\end{tabular}




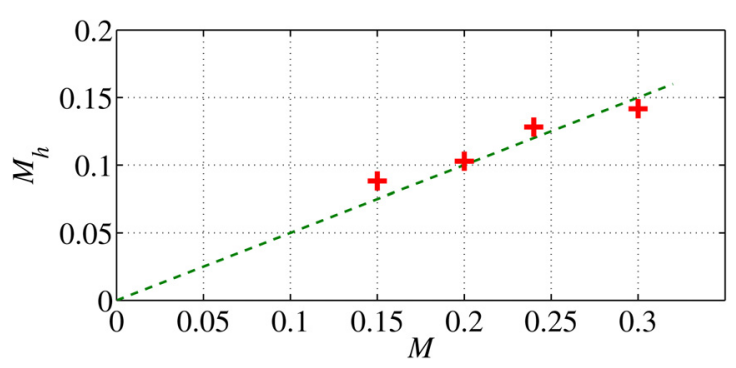

FIG. 9. (Color online) Normalized velocity of the hydrodynamic wave $M_{h}=c_{h} / c_{0}$ as a function of mean flow Mach number $M$. (134 dB SPL). The straight line corresponds to $M_{h}=0.5 M_{0}$.

\section{MICROPHONES MEASUREMENTS ON THE WALL OPPOSITE TO THE POROUS MATERIAL}

The transmission coefficient described in the previous section links the incident plane wave at $x=0$ to the outcoming plane wave at $x=L$ when the downstream duct is supposed to be anechoic. To gain a deeper insight of what happens between the entrance $x=0$ and the exit $x=L$ and validate the scenario described previously, the measurements of the array of 11 flush mounted microphones located on the wall opposite to the porous material are used.

Once again, these measurements will be fitted taking into account the above scenario with a minimum of new parameters. The two waves are supposed to travel between $x=0$ and $x=L$ with a constant velocity. The acoustic velocity is frequency dependent [see Fig. 8(b)] and the value used in the following is the previously determined value. To fit the $x$-dependence of the amplitude of the acoustical wave, it is coherent to use also an exponential behavior $p_{a}^{+}(x) / p_{1}^{+}$ $=e^{-j k_{a} x}$. Again, the imaginary part of $k_{a}$ is the previously determined value. Then the $x$-dependence of the acoustic part is supposed to be completely determined by the knowledge obtained from the analysis of $T^{+}$.

The hydrodynamic part is supposed to have a phase equal to $\theta_{0}+\omega L / c_{h}$ where $c_{h}$ is the velocity of the hydrodynamic wave previously determined and $\theta_{0}$ is the phase lag between acoustic and hydrodynamic waves determined by using the value for $f=0$ in Fig. 9(b). The value $\theta_{0}=-0.3 \pi$ will be used in the following. The only quantity that has not been determined previously is the $x$-dependence of the amplitude of the hydrodynamic wave. This quantity cannot be described by an exponential behavior because of the non-linear saturation.
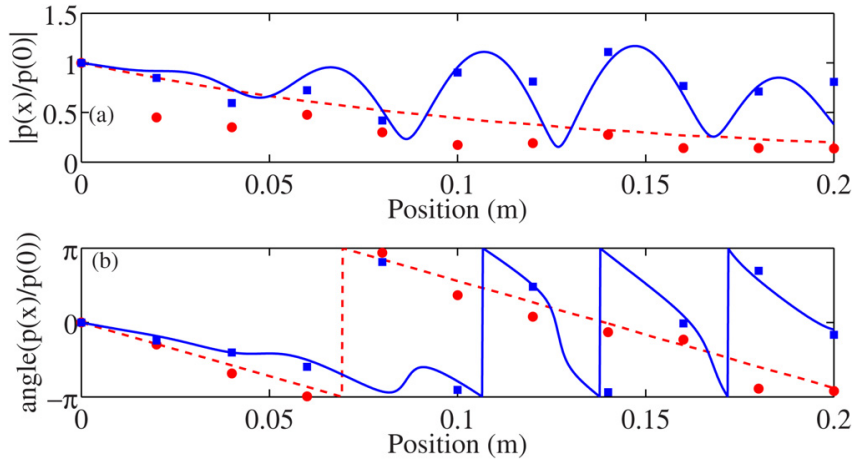

FIG. 10. (Color online) Pressure normalized by the pressure at $x=0$ on the wall opposite to the porous material ( $M=0.3$ and $134 \mathrm{~dB}$ SPL) given in amplitude (a) and phase (b). $f=2500 \mathrm{~Hz}$ circle: measurements, dashed line: fitted. $f=1600 \mathrm{~Hz}$, square: measurements, continuous line: fitted.

A simple polynomial fitting $h(x)=a(X / L)^{2}-(a-1)(X /$ $L)^{4}$ is used with $a=30$.

Accordingly, the pressure on the $p(x)$ normalized by the pressure at $x=0$ is written as

$$
\frac{p(x)}{p(0)}=e^{-j k_{a} x}+H h(x) e^{-j\left(\theta_{0}+\omega x / c_{h}\right)},
$$

where $H$ is the amplitude of the hydrodynamic transmission [Fig. 8(a)]. It should be emphasized that the above expression is not a model of the behavior of the porous liner with grazing flow but just a way to fit and understand the measurements.

A comparison between the measured pressure and the values given by Eq. (1) is plotted in Fig. 10. It can be seen that the agreement is reasonable in phase when the hydrodynamic wave is absent $(f=2500 \mathrm{~Hz}$, circles $)$ and it can be easily improved by taking into account the entrance and exit effects and the reflected waves on the material zone. When the hydrodynamic wave is present $(f=1600 \mathrm{~Hz}$, squares), the spacing between the microphones $(20 \mathrm{~mm})$ is too large to give a precise outline of the hydrodynamic wave $\left(\lambda_{h}\right.$ $\simeq 35 \mathrm{~mm}$ ). Again the phase agreement is reasonable. Nevertheless, the 2D plot in Fig. 11 depicting the real part of the pressure as a function of position and frequency shows that the main tendencies are described by the fit. In particular, the isolines in white in Fig. 11, representing the condition $\omega x / c_{h}=2 n \pi$, confirm that the hydrodynamic wave is

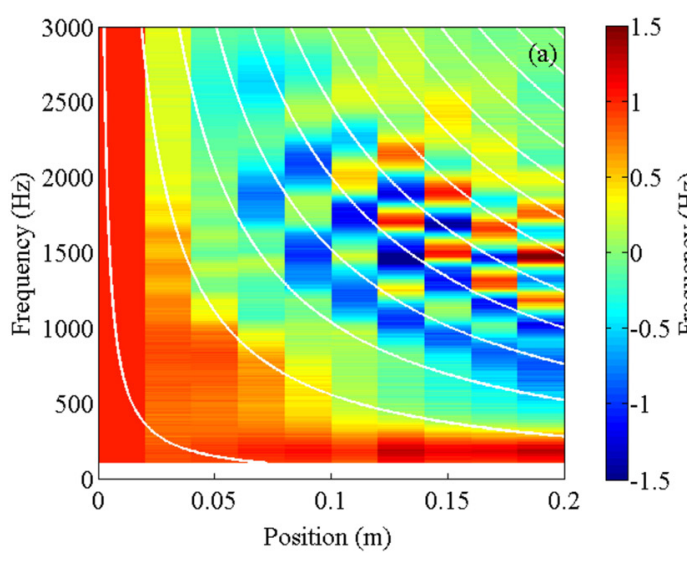

J. Acoust. Soc. Am., Vol. 136, No. 2, August 2014

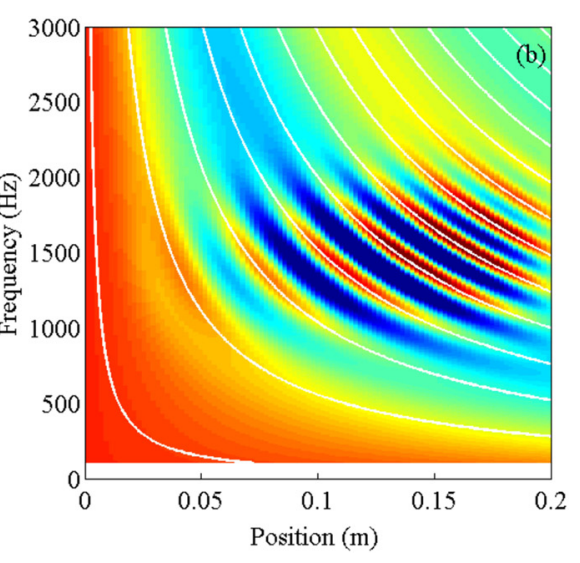

FIG. 11. (Color online) Measured (a) and fitted (b) pressure (normalized by the total pressure at $x=0$ ) on the wall opposite to the porous material for $M=0.3$ and $134 \mathrm{~dB}$ SPL. The source is located upstream and the effect of the downstream reflection (downstream of the lined section) has been removed using the two sources method. The white lines represent the condition: $\omega x / c_{h}=2 n \pi$. 

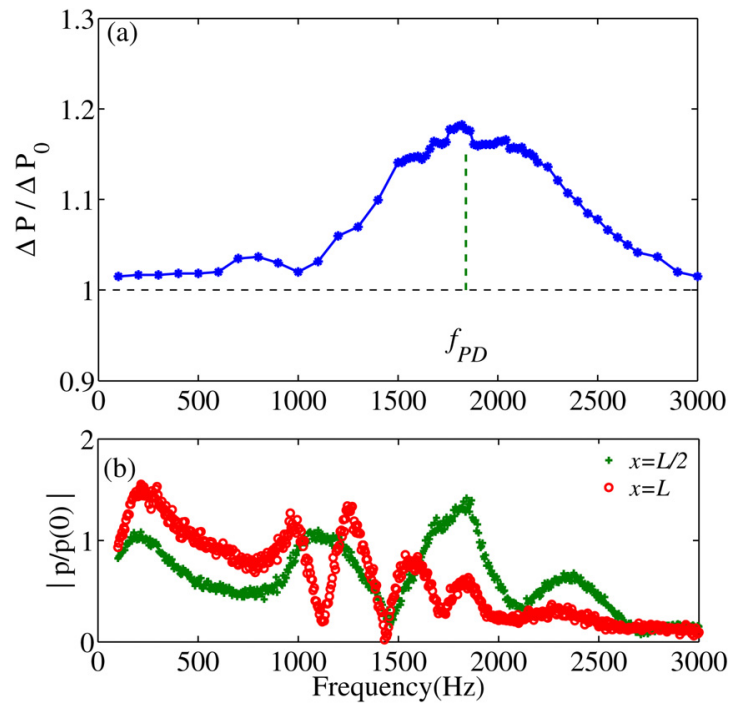

FIG. 12. (Color online) (a) Pressure drop with sound $\Delta P$ normalized by the pressure drop without sound $\Delta P_{0}$ as a function of the incident frequency. (b) Absolute value of the normalized pressure on the wall opposite to the porous material for two positions. $M=0.3$ and $134 \mathrm{~dB}$ SPL.

convected with velocity $c_{h}$. However, the progressive shifting of the experimental maxima relatively to the maxima of the fit suggests that the convection velocity of the hydrodynamic wave is not constant along the material.

\section{PRESSURE DROP ALONG THE POROUS MATERIAL}

It is known that a convected instability can induced an increase of the static pressure drop along the material. ${ }^{4,8}$ This added dissipation is due to an increase of the turbulent wall shear stress induced by the hydrodynamic wave. ${ }^{5,9} \Delta P_{0}$ is the static pressure difference between the start and the end of the porous material without any acoustic excitation. For this porous material, $\Delta P_{0}$ increases quadratically as a function of Mach number. $\Delta P$ is the same static pressure difference when the sound source is on. Figure 12(a) shows the increase in pressure drop when the acoustic source is turned on. It has been measured that the pressure drop increase is nearly proportional to the incident wave pressure amplitude above a threshold level of $120 \mathrm{~dB}$ SPL. The relative increase is of the order of $20 \%$ to $40 \%$ whatever the Mach number for a source level equal to $134 \mathrm{~dB}$ SPL. This increase is much smaller than the one observed in locally reacting liners. ${ }^{4}$

When the pressure drop is plotted against frequency (Fig. 12), it is expected that the frequency at which the pressure drop is maximum $f_{\mathrm{PD}}$ corresponds to the frequency at which the amplitude of the hydrodynamic wave is maximum $f_{m}$. It can be seen in Table I that these frequencies are not equal. A possible explanation of this phenomena is that frequency range where the hydrodynamic wave has a large amplitude is bigger for $x \simeq L / 2$ than at the end of the material $x=L$ [see Figs. 12(b) and 11(a)]. It is unclear, at this point, whether this corresponds to an attenuation of the hydrodynamic wave in the region $L / 2<x<L$ or to a diminution of the pressure induced by the hydrodynamic wave in this region. Further studies with optical measurements (particle image velocimetry or laser Doppler velocimetry) are needed to clarify this point.

\section{CONCLUSION}

The existence of a hydrodynamic wave over a porous material with grazing flow has been demonstrated. Its characteristic properties have been analyzed experimentally. This hydrodynamic wave has a significant effect on a limited band of frequencies. When this wave is present, there are large oscillations in the transmission coefficient in the flow direction coming from the interference between the transmitted acoustic wave and this hydrodynamic wave that are propagating at different velocities. The convection velocity of the hydrodynamic wave is close to half of the mean flow speed. It is difficult to claim any firm conclusions about the amplitude of this mode because of the non-linear behavior that indicates a saturation of this hydrodynamic wave. The hydrodynamic wave induces an increase of the pressure drop when it is created by an acoustic wave. A peak frequency at which the pressure drop is maximum is identified. Further investigations are needed to model the conditions of appearance, amplification, and saturation of this new kind of hydrodynamic wave.

\section{ACKNOWLEDGMENTS}

We gratefully acknowledge the support from the European Union through ITN-project FlowAirS (Contract No. FP7-PEOPLE-2011-ITN-289352).

${ }^{1}$ D. L. Johnson, J. Koplik, and R. Dashen, "Theory of dynamic permeability and tortuosity in fluid-satured porous media," J. Fluid. Mech. 176, 379-402 (1987).

${ }^{2}$ D. Lafarge, P. Lemarinier, J. F. Allard, and V. Tarnow, "Dynamic compressibility of air in porous structures at audible frequencies," J. Acoust. Soc. Am. 102, 1995-2006 (1997).

${ }^{3}$ J. F. Allard and N. Atalla, Sound Propagation in Porous Materials Having a Rigid Frame, 2nd ed. (Wiley and Sons, Chichester, UK, 2009), Chaps. 5 and 6.

${ }^{4}$ Y. Aurégan and M. Leroux, "Experimental evidence of an instability over an impedance wall in a duct with flow," J. Sound Vib. 317, 432-439 (2008).

${ }^{5}$ D. Marx, Y. Auregan, H. Bailliet, and J. C. Valiere, "PIV and LDV evidence of hydrodynamic instability over a liner in a duct with flow," J. Sound Vib. 329(18), 3798-3812 (2010).

${ }^{6} \mathrm{~S}$. W. Rienstra, "A classification of duct modes based on surface waves," Wave Motion 37, 119-135 (2003).

${ }^{7}$ Y. Renou and Y. Aurégan, "Failure of the Ingard-Myers boundary condition for a lined duct: An experimental investigation," J. Acoust. Soc. Am. 130(1), 52-60 (2011).

${ }^{8} \mathrm{M}$. Brandes and D. Ronneberger, "Sound amplification in flow ducts lined with a periodic sequence of resonators," in the 1st AIAA/CEAS Aeroacoustics Conference, AIAA 95-126 (1995), pp. 893-901.

${ }^{9} \mathrm{D}$. Ronneberger and M. Jüschke, "Sound absorption, sound amplification, and flow control in ducts with compliant walls," in Oscillations, Waves and Interactions, edited by T. Kurz, U. Parlitz, and U. Kaatze (Universitätsverlag Göttingen, Göttingen, 2007), pp. 73-106. 